\title{
Binary Stars in the Local Group: The Playing Field
}

\author{
Mario L. Mateo \\ Department of Astronomy, University of Michigan, 821 Dennison \\ Building, Ann Arbor, MI 48109-1090, USA
}

\begin{abstract}
As an introduction to the stage on which Extragalactic Binaries exist I will present an overview of the stellar populations of the Local Group Galaxies. This will highlight the (few) similarities in the star formation histories of Local Group galaxies as well as the better-known variety that has been uncovered in recent studies. In the few cases where this is possible, the direct role of binaries in the interpretation of the star forming histories of these galaxies will be described. The possible role of binaries in helping to shape the star-formation history will be described more generally with some emphasis on the sorts of observations that can be carried out to investigate this more fully.
\end{abstract}

